\title{
HISTORIA EMPRESARIAL \\ E HISTORIA DEL TRABAJO: \\ DEL AISLAMIENTO A LA COLABORACIÓN
}

\author{
JOSÉ IGNACIO MARTÍNEZ RUIZ \\ Universidad de Sevilla
}

Esta nota pretende llevar el debate abierto en torno al tema de qué historia empresarial podemos y queremos hacer, más allá del marco en el que se viene desarrollando hasta el presente ${ }^{1}$. En este sentido, propugna como idea central que las agendas de investigación y la docencia de historia empresarial incorporen las reflexiones y resultados obtenidos por los historiadores del trabajo en los últimos años ${ }^{2}$.

La oportunidad del acercamiento entre ambas disciplinas viene sugerida fundamentalmente por: a) la profunda renovación que han conocido los estudios de historia del trabajo desde finales de los años cincuenta o comienzos de los sesenta, sobre todo en Gran Bretaña y Estados Unidos, cuyas historiografías constituyen el principal punto de referencia bibliográfico de estas páginas 3; renovación que apenas ha encontrado eco entre los historiadores económicos y de la empresa de nuestro país, y b) las excelentes expectativas y, en algún caso ya, resultados, que ofrece la colaboración entre los historiadores de la empresa y los historiadores del trabajo ${ }^{4}$.

Así las cosas, en las páginas que siguen expondremos en primer lugar los cambios más significativos que han afectado a la historia del trabajo como área

1 Coll (1991), Coll y Tortella (1992), Valdaliso (1993), Torres Villanueva (1993).

2 De igual forma, propugnamos que las agendas de investigación y la docencia de historia del trabajo incorporen las reflexiones y resultados obtenidos por los historiadores de la empresa en los últimos años.

3 Sobre Alemania, véase Saldern (1994).

4 Ross y Perkins (1986). 
de estudio desde su aparición hacia el cambio de siglo hasta el presente (I); a continuación analizaremos, tomando como ejemplo concreto la cuestión del establecimiento de la «empresa moderna» en Estados Unidos y Gran Bretaña, lo que la historia del trabajo puede aportar a un mejor conocimiento de temas como éste, un «clásico» de la reciente historia empresarial (II); y concluiremos con una breve reflexión de indole general (III).

Los origenes de la historia del trabajo como disciplina académica se remontan a los años finales del siglo xIx y primeros del XX. En el caso de Gran Bretaña, el punto de referencia más importante en tal sentido lo constituye la publicación por Sidney y Beatrice Webb [fundadores de la London School of Economics en 1895] de «A History of Trade Unionism» el año 1894, estudio en el que la materia se identifica con la historia de las

asociación(es) permanente(s) de trabajadores por cuenta ajena con la finalidad de mantener o mejorar las condiciones de su vida de trabajo ${ }^{5}$.

Al otro lado del Atlántico, en los Estados Unidos, los primeros pasos de la historia del trabajo, entendida también como historia de las organizaciones obreras, aparecen asociados al empeño de algunos economistas de formación, fuertemente influidos por la escuela histórica alemana, entre quienes sobresale John R. Commons (diversas publicaciones, a destacar, junto con otros autores, "The History of Labor in the United States", Nueva York, 1918, dos primeros volúmenes) y, de manera más general, a la denominada «Escuela de Wisconsin», de la que fueron también insignes representantes Selig Perlman («A Theory of the Labor Movement», Nueva York, 1928) y Philip Taft ${ }^{6}$.

Las críticas recibidas por esta «primera» historia del trabajo a partir de finales de los años cincuenta o comienzos de los sesenta, a las que en seguida nos referiremos por extenso, y que en buena medida se explican por el interés del movimiento renovador en marcar las distancias con lo que se quería dejar atrás, no pueden llevarnos a ignorar algunos de sus logros y peculiaridades más significativos: tanto en Gran Bretaña como en Estados Unidos, la «vieja» historia del trabajo reivindicó un nuevo objeto de atención, proporcionó las

s Webb, S. y B. (1990) [1920], p. 21.

- Sobre los origenes de la historia del trabajo en los Estados Unidos, véase el articulo reivindicativo de Fink (1991), quien sitúa los primeros estudios sobre el tema en la década de 1880 . 
primeras sintesis en torno al mismo y estableció las bases documentales sobre las que se erigirian buena parte de las investigaciones posteriores ${ }^{7}$; asimismo, su carácter reformista (circunstancia que explica la participación de las personalidades más destacadas de la «Escuela de Wisconsin» en la agitada vida polí. tica norteamericana del período y la adscripción de los Webb al socialismo fabiano) y empírico (frente al excesivo formalismo de los economistas neoclásicos), constituyó un soplo de aire fresco en un ambiente cargado de conservadurismo social y académico ${ }^{8}$.

Al carácter esencialmente institucional de esta primera historia del trabajo se opone, a partir de finales de los años cincuenta o comienzos de los sesenta ${ }^{9}$, una nueva concepción de la misma, tarea que protagonizan, entre otros, $\mathrm{E}$. P. Thompson ${ }^{10}$ y E. Hobsbawm ${ }^{11}$ en Gran Bretaña, donde se configura una «historia social del trabajo» ${ }^{12}, \mathrm{y}$, fuertemente influidos por los anteriores, $\mathrm{H}$. Gutman ${ }^{13}$ y D. Montgomery ${ }^{14}$ en Estados Unidos, donde tiene lugar el nacimiento de una «nueva historia del trabajo».

La renovación emprendida afecta tanto al objeto como al método utilizados por los primeros historiadores del trabajo ${ }^{15}$. El objeto de la disciplina, efectivamente, se amplía: entendida como historia de las organizaciones obreras, la primera historia del trabajo había privado a la mayor parte de la población laboral, la no sindicada -mucho más numerosa en el caso de los Estados

7 Como «A Documentary History of American Industrial Society» de Commons y otros (Cleveland, 1910-1911, 10 vols.).

8 Brody (1993), pp. 4-6.

- El año 1960 aparece el primer número de la revista norteamericana «Labor History», a la que seguiria poco después «International Labor and Working Class History». En 1960 también se crea en Gran Bretaña la «Sociedad para el Estudio de la Historia del Trabajo». Otros aconte cimientos de trascendencia para la disciplina y situados cronológicamente a finales de los años cincuenta o comienzos de los sesenta, en Zieger (1983, pp. 61-63).

10 Thompson [1963].

11 Hobsbawm (1979) [1964]

12 Un importante precedente de esta renovación lo constituyen, para el caso británico, los es posos Hammond («El trabajador del campo», Londres, 1911; «El trabajador de la ciudad», Lon dres, 1917, y «El trabajador especializado», Londres, 1919). Véase, en este sentido, Fontana (1987.88), p. 147.

13 Diversos articulos y comunicaciones a congresos a partir de la lectura de su tesis doctoral el año 1961, entre los que sobresale Gutman (1973).

14 Montgomery (1967) y (1985) [1979]. Se trata de una recopilación de articulos publicados en diversas revistas entre los años 1974 y 1978, comenzando por el que da título al libro «El control obrero de la producción industrial en el siglo $\mathrm{XIX}_{\text {\% }}$ (aparecido inicialmente en «Labor History», otoño de 1976).

15 Sobre las diferencias entre la «vieja» y la «nueva» historia del trabajo norteamericanas versan los artículos de Kreuger (1971), tal vez el primero en utilizar esos calificativos, Brody (1979) y Montgomery (1980). La evolución de la historia del trabajo en Gran Bretaña se puede seguir en Zeitlin (1987). 
Unidos, país en el que a comienzos de la década de 1930 suponía aún cerca del $90 \%$ de la población activa no agrícola, que en Gran Bretaña-, de toda posibilidad de un estudio serio y riguroso ${ }^{16}$. Asimismo, al poner el acento en los líderes y las burocracias sindicales, el protagonismo de los trabajadores anónimos y de los militantes de base había resultado totalmente desdibujado. Se comprende asi que en unos años, los sesenta, caracterizados por el hecho de que los sindicatos y la negociación colectiva estaban fuertemente consolidados en todo el mundo occidental, como parte esencial que eran de la política de consenso social establecida al término de la Segunda Guerra Mundial, los historiadores del trabajo comenzaran a formularse nuevas preguntas, relacionadas ahora, más que con las claves institucionales y políticas de la historia del movimiento obrero, con las de los trabajadores como tales.

Dado, de otra parte, que la historia del trabajo se venia desarrollando al amparo y bajo las coordenadas que marcaba su vinculación al análisis económico, sobre todo en Estados Unidos, circunstancia que habia provocado un significativo aislamiento de la misma con respecto a la evolución seguida por otras disciplinas históricas, como la historia social o cultural, se comprende también que los nuevos historiadores del trabajo llevaran a cabo una decidida reivindicación de la cultura y la experiencia en el trabajo como elementos que nuclean la identidad y la fuerza de clase obrera ${ }^{17}$.

Asi lo expresaba E. Hobsbawm en el Prefacio al conjunto de artículos que, bajo la denominación de "Trabajadores», aparecian en forma de libro el año 1964:

La mayoría de estos articulos tienen en común un rasgo negativo: se sitúan al margen de la historia directamente cronológica o narrativa de los movimientos obreros. Sidney y Beatrice Webb y G. D. H. Cole fueron los talentosos precursores de este enfoque y en la edad de oro de la historia del movimiento obrero británico, que se inició hace unos quince años, una serie de excelentes estudios prosiguieron, completaron o revisaron su obra. Sin embargo, se ha investigado comparativamente poco acerca de las clases trabajadoras como tales (no en cuanto organizaciones y movimientos obreros) y acerca de las condiciones económicas y técnicas que favorecieron el desarrollo efectivo de los movimientos obreros o bien, en cambio, lo dificultaron. (...) ${ }^{18}$.

16 Gutman (1973), p. 536.

17 A pesar de su común oposición a la «vieja», no se puede ignorar que dentro de la «nueva» historia del trabajo norteamericana conviven, al menos, dos tendencias: la primera, centrada en el estudio de la cultura obrera (Gutman); la segunda, interesada en las luchas que se mantuvieron en las fábricas por el control de la organización de la producción (Montgomery). Una amplia y critica exposición de estas tendencias, en McDonnell (1991) [1984].

18 Hobsbawm (1979) [1964]. El más antiguo de estos articulos fue publicado en 1957. 
En términos similares se expresaría Gutman, diez años después, en un artículo señero, por su carácter programático, para los seguidores de la nueva orientación en Estados _Unidos:

las páginas que siguen prestan poca atención a los temas que se consideran propios de la historia del trabajo (sindicatos, huelgas y movimientos radicales) ${ }^{19}$.

Por lo que hace a los fundamentos teóricos y metodológicos de la historia social del trabajo británica o de la nueva historia del trabajo norteamericana, debemos resaltar que la renovación de la que estamos hablando se lleva a cabo en medio de una significativa precariedad, por lo que al análisis económico se refiere, y aun de una marcada hostilidad hacia la economía del trabajo. ¿Por qué? De un lado, conviene tener en cuenta que los protagonistas del proceso en Gran Bretaña (Hobsbawm, Thompson, etc.) trataban de superar el economicismo legado por el marxismo vulgar, que tan ampliamente se había difundido desde finales del siglo XIX, $y$, más recientemente, por el estructuralismo marxista de corte althusseriano (alternativa: «la historia desde abajo»); de otro, que la «nueva» historia del trabajo norteamericana habia surgido en oposición a la «vieja» escuela institucional (Commons, «Escuela de Wisconsin»), donde la historia y la economía del trabajo aparecian plenamente integradas.

Pero, al margen de esto, ¿qué podía ofrecer hacia 1960 la economía del trabajo, en su doble vertiente de disciplina volcada en el estudio del mercado de trabajo y de las relaciones industriales a la historia del trabajo? Según G. Wright, la economía del trabajo se habia convertido en Estados Unidos, y en oposición también a la «escuela institucional», en una rama ahistórica de la economía aplicada ${ }^{20}$, merced a su progresiva integración, a partir de los precedentes de A. C. Pigou («Wealth and Welfare», 1912) y J. R. Hicks («Theory of Wages», 1932), en el aparato analítico neoclásico, del que la teoría del capital humano de la «Escuela de Chicago» constituia por entonces su producto más elaborado ${ }^{21}$. Por lo que hace al estudio de las relaciones industriales, hacia 1960 también se encontraba ampliamente difundida una visión un tanto ecléctica dominada por los trabajos de Kerr y Dunlop en los Estados Unidos y de la «Escuela de Oxford» en Gran Bretaña, en la que, pese a las llamadas en fa-

19 Gutman (1973), p. 537.

20 Wright (1987), p. 315.

21 Mcnulty (1980, pp. 177 y siguientes). Toharia (1983), «Introducción», para un resumen de los rasgos que caracterizan a las distintas escuelas de pensamiento económico sobre el mercado de trabajo: neoclásica, marxista e institucional. Un reciente estado de la cuestión, en Rebitzer (1993). 
vor de un análisis histórico de las relaciones industriales ${ }^{22}$, se había impuesto también una visión atemporal y cerrada de las mismas. El libro de Kochan sobre la experiencia norteamericana constituye una de sus mejores expresiones ${ }^{23}$. Los economistas del trabajo, por su parte, tampoco parecían dispuestos a considerar los cambios habidos en la historia del trabajo y a incorporar los resultados obtenidos por sus investigadores ${ }^{24}$.

La importante renovación que conoce la historia del trabajo a partir de finales de los años cincuenta o comienzos de los sesenta se lleva a cabo, asimismo, al margen de la que por entonces estaba teniendo lugar en el marco de la historia empresarial, con los trabajos de Chandler como expresión más sobresaliente ${ }^{25}$. ¿Por qué? Habria que destacar, tal vez, dos circunstancias: la primera, que tradicionalmente una y otra disciplinas habian mantenido inclinaciones ideológicas distintas. Los historiadores de la empresa, efectivamente, aparecian como los defensores del libre funcionamiento del mercado y de la institución empresarial; los historiadores del trabajo, y especialmente la «nueva» corriente (izquierdista y radical frente al «reformismo» de la «Escuela de Wisconsin»), como sus críticos. Por otra parte, los intereses teóricos y el «sentido» de la renovación de una y otra disciplinas eran justamente contrarios: la historia empresarial «tradicional» aparece volcada hacia el estudio de hombres de negocios y compañías concretos, constituyendo el «caso-estudio» el modelo de investigación predominante. $\mathrm{La}$ «nueva» historia empresarial, por contra, reacciona frente al excesivo empirismo de la anterior, de ahí dos de sus rasgos más importantes: el reforzamiento del bagaje teórico que le sirve de fundamento y su carácter comparado. En el caso de la historia del trabajo, mientras tan-

22 Nos referimos a Dunlop (1978) [1958] y Kerr (1964). Con respecto a tales llamadas, véase, por ejemplo, el capitulo VIII del libro de Dunlop, donde se explicita que «la perspectiva histórica y una apreciación del cambio a través del tiempo son esenciales para la teoría de las relaciones industriales» (p. 289), o el capitulo X, donde se afirma que «el concepto de sistema de relaciones industriales se emplea de forma más fructifera como herramienta de anälisis cuando se examina un determinado sistema en su contexto histórico y se estudian los cambios en el sistema a lo largo del tiempo» (p. 358).

${ }^{23}$ Kochan (1980). M. Detber, uno de los intervinientes en el «review symposium» sobre este libro, publicado por Industrial Relations (vol. 21, n. 1, 1982), afirma explícitamente: «[en esta obra] no hay reflexión alguna sobre el debate que se ha producido en los últimos doce años entre los historiadores americanos dedicados al estudio de la cultura de la clase trabajadora y los defensores de la Escuela de Wisconsin, centrados en el estudio de las organizaciones obreras y la negociación colectiva" (p. 86). Otro de los participantes en el symposium, R. Hyman, se refiere a la existencia de una visión alternativa, marxista, de las relaciones industriales a la defendida por Kochan, uno de cuyos rasgos caracteristicos seria la sensibilidad mostrada hacia las dimensiones temporales de los fenómenos (p. 108).

24 Ozanne (1980), uno de los últimos herederos de la «Escuela de Wisconsin», se lamenta de la «deserción» de los economistas del trabajo hacia la historia del trabajo (p. 513).

25 Chandler (1962), Chandler (1987) [1977]. 
to, se estaba siguiendo el camino justamente inverso: la «nueva» historia del trabajo perfila sus señas de identidad en oposición a las que se consideran generalizaciones abusivas y simplificadoras de la «vieja». Dadas estas circunstancias, difícilmente podia producirse un intercambio teórico y metodológico entre ambas, permaneciendo una y otra disciplinas en una situación de mutuo aislamiento, extensible también a la historia económica «general» ${ }^{26}$.

En los ültimos años, sin embargo, algo está empezando a cambiar. Del lado de la economía del trabajo, la insuficiencia manifestada por la corriente principal para explicar el funcionamiento del mercado de trabajo, ha dado pie al desarrollo de nuevos planteamientos, como la teoría de la «balcanización» de los mercados de trabajo ${ }^{27}$, de la segmentación del mercado de trabajo ${ }^{28}$, o de la pérdida de cualificación del trabajo que caracterizaría el desarrollo capitalista en su fase monopolista ${ }^{29}$, planteamientos en los que se han inspirado, siquiera parcialmente, importantes estudios con una clara orientación histórica como los de Gordon, Reich y Edwards ${ }^{30}$ o Piore y Sabel ${ }^{31}$.

En otro orden de cosas, la crisis de los setenta y ochenta, sobre todo en Estados Unidos, donde se produce por entonces un importante descenso del indice de afiliación sindical, una clara revisión de los supuestos del consenso sobre el papel que tocaba desempeñar a cada una de las partes en juego en que se habian basado las relaciones laborales desde el New Deal y el desarrollo de sistemas alternativos de gestión de los recursos humanos, ha llevado a replantear la visión de las relaciones industriales de postguerra como un sistema cerrado y a valorar el carácter contingente de las mismas, circunstancia que ha exigido considerar de nuevo su evolución histórica. En este sentido basta comparar los trabajos de síntesis publicados por Kochan en 1980 y $19899^{32}$, por lo que hace al estudio de las relaciones industriales en los Estados Unidos,

26 En la «Introducción» del libro, Jacoby (1991) amplía el argumento que mantenemos aqui sobre las vinculaciones mantenidas por la historia del trabajo y la historia empresarial a la histolas mismas conclusiones (p. 4). Durante las décadas de 1960 y 1970 , efectivamente, los económetras estadounidenses mantuvieron unas relaciones altamente impermeables con los historiadores del trabajo y de la empresa, a los que prácticamente ignoraron, y viceversa.

27 Kerr (1950).

28 Piore y Doeringer (1971).

29 Braverman (1974)

30 Gordon, Edwards y Reich (1986) [1982].

31 Piore y Sabel (1990) [1984].

32 Kochan (1980), Kochan, Katz y McKersie (1993) [1989]. El segundo capitulo de éste tiene como tema la «Evolución histórica del sistema de negociación colectiva en los Estados Unidos» (a partir del New Deal). 
y por Palmer en 1983 y $1993^{33}$, por lo que hace a Gran Bretaña, para apreciar el largo camino recorrido.

Los cambios económicos habidos en los años 70 y 80 han sugerido, igualmente, a los estudiosos del trabajo y de las relaciones industriales una mayor atención hacia aquellas publicaciones de historia empresarial consagradas al examen de los problemas planteados por la gestión. En palabras de uno de ellos:

En la medida en que la investigación sobre las relaciones laborales considere los efectos de las decisiones económicas estratégicas, podrá beneficiarse de las actuales investigaciones sobre la politica y estrategia de las empresas. La obra clásica de Alfred Chandler sobre la relación entre estrategia y estructura puede ayudar a comprender la evolución de la estructura de gestión y de las políticas de relaciones laborales dentro de la empresa ${ }^{34}$.

¿Qué movimientos se observan, mientras tanto, entre los historiadores del trabajo? Tras la celebración de sus éxitos durante las décadas de 1960, 70 y $80^{35}$, se abre la cuestión de qué camino seguir y de las tareas prioritarias que conviene acometer. Entre las propuestas barajadas por las figuras más representativas de la disciplina se encuentran: a) sintetizar el enorme esfuerzo de los últimos años en alguna obra que aglutine el estado actual de los conocimientos sobre el tema ${ }^{36}$. Esta viene siendo una de las principales preocupaciones de los historiadores del trabajo norteamericanos desde que Brody (1979, pp. 2426) sugiriera la necesidad de reemplazar los viejos textos de Commons y Perlman; b) proceder a una reconciliación con la «vieja» historia del trabajo, una vez olvidado el fragor de la batalla que dio lugar al nacimiento de la «nueva» ${ }^{37}$. De forma implicita, el último libro de Montgomery (1987) ha abierto el camino a una revisión de algunos de los supuestos en los que se había basado tradicionalmente la «nueva» historia del trabajo, pues en él se proponen, entre

${ }^{3}$ Palmer (1983), Palmer y Gospel (1993).

34 Kochan, Katz y McKersie (1993) [1989], p. 33

3" «Los últimos veinte años han sido una verdadera edad de oro de la historia de la clase obrera», escribe Hobsbawm (1987) [1984], «Prefacio».

${ }^{36}$ Una crítica del trabajo de Gordon, Edwards y Reich como «primera interpretación general» de la historia de la clase obrera norteamericana desde los textos de Commons y Perlman, en Schatz (1984).

37 Esta es la propuesta de que hace Brody (1993) tras constatar dos hechos: que la "vieja" historia del trabajo no ha sido completamente desplazada por la «nueva», como lo demuestran los temas de los artículos que pueblan las principales revistas del ramo u otro tipo de publicaciones academicas, y que la unidad de perspectiva que caracterizó en sus comienzos a la «nueva» historia del trabajo ya no existe (el autor habla de «cacofonía de prescripciones acerca de hacia donde debe dirigirse» la disciplina). 
otras cosas, recuperar el protagonismo de las «minorías militantes» (frente a la «historia desde abajo»), considerar nuevamente el papel del Estado (frente al olvido de la «política») o valorar suficientemente lo que supuso la desaparición del capitalismo competitivo clásico; c) llevar a cabo un rearme teórico ${ }^{38}$ que, inevitablemente, traería consigo un acercamiento a la economía del trabajo $\mathrm{y}$, mejor aún, a la historia de la empresa y a la historia económica ${ }^{39}$, así como una clara apuesta en favor de los estudios comparados ${ }^{40}$, y d) refundar la historia del trabajo, ahora como historia de las relaciones industriales, esto es, de las cambiantes relaciones entre trabajadores, sindicatos, patronal y Estado ${ }^{41}$.

El establecimiento de la «moderna empresa gerencial» constituye, de la mano de A. Chandler (1977 y 1990), uno de los temas estelares de la reciente historia empresarial. En los libros más representativos de éste, sin embargo, no se hallará prácticamente ninguna referencia al mercado de trabajo o al sistema de relaciones industriales característicos de los paises objeto de estudio ${ }^{42}$. ¿Puede ignorarse una cuestión como ésta a la hora de explicar el triunfo del sistema de producción en serie, mediante el empleo de maquinaria altamente especializada y de trabajadores no cualificados que caracteriza la experiencia industrial norteamericana del siglo XIX, o la supervivencia de formas de pro-

38 Brody (1989) plantea en este artículo la conveniencia de renovar los lazos que tradicional. mente habian mantenido la historia y la economía del trabajo en el seno de la «vieja escuela institucional», pero no sobre las bases de antaño. A su juicio la historia del trabajo no puede ser un mero apéndice de la economía del trabajo y debe permanecer en el seno de la comunidad constituida por los historiadores. Este nuevo maridaje exige que la historia del trabajo sea tomada en serio y se consideren sus aportaciones; en sentido contrario, el gran reto para una historia del trabajo consciente de sus, con frecuencia, estrechos marcos de investigación -el marco local-y de la complejidad y variedad de las experiencias de la clase obrera de ahí la atención prestada a temas como la raza, religión, familia, movilidad, etc. --es la revisión de las categorías analíticas utilizadas.

39 Esta es la propuesta de Wright (1987), para quien la alternativa a la ortodoxia neoclásica sobre el trabajo no es un nuevo institucionalismo sino la historia económica (p. 319).

40 «Se requiere una nueva apreciación de la naturaleza internacional del conflicto laboral Esto será impulsado por más estudios comparativos», sugiere McDonnell (1991), p. 97. La histo ria del trabajo británica ha sido demasiado insular; debemos añadir la dimensión comparativa a lo que hacemos, reconoce Price (1991), p. 259.

41 Zeitlin (1987), pp. 159 y 178. Frente a ésta se encuentra la propuesta de Price (1991), para quien la disciplina debe retomar como cuestión central el papel jugado por el trabajo en la conformación del conjunto social, es decir, debe continuar siendo una historia social del trabajo.

42 Si se encuentran, por el contrario, en el «manual» utilizado por el autor en la Harvard Business School (Chandler y Tedlow 1985), que dedica cuatro lecciones al tema. 
ducción «artesanales» y basadas en la utilización de una abundante mano de obra cualificada, circunstancias ambas que parecen haber acelerado el declive del sector industrial, propia del modelo británico? Pensamos que no; y que esta circunstancia nos impide disponer de una versión más completa sobre la verdadera dinámica del capitalismo industrial y sobre las razones de la diversidad de las experiencias y de los éxitos o fracasos económicos nacionales.

El predominio del sistema de producción artesanal en Gran Bretaña (y, con él, de la pequeña empresa dirigida por sus propietarios) se ha explicado tradicionalmente porque la creación de una administración gerencial compleja y la realización de grandes inversiones en maquinaria especializada sólo tienen sentido si existe un mercado amplio y dinámico para productos estandarizados. Este no era el caso de Gran Bretaña a finales del siglo XIX, donde los mercados de productos eran muy competitivos y se encontraban muy fragmentados. Pero, igualmente, a la hora de explicar la supervivencia del sistema de fabricación artesanal no podemos olvidar que en presencia de una abundante mano de obra cualificada como ocurria en Gran Bretaña, muchas empresas optaron por seguir utilizando métodos tradicionales de producción antes que por introducir innovaciones tecnológicas que requerian fuertes inversiones y un mayor número de trabajadores no cualificados. En bastantes sectores, estas consideraciones pudieron haber obstaculizado el proceso de modernización y racionalización industrial, con los efectos de todos conocidos. De otra parte, la existencia en Gran Bretaña de uno de los movimientos sindicales más poderosos del mundo conllevó también que los empresarios encontraran una gran resistencia a la introducción de nuevos métodos de producción y sistemas organizativos en aquellos casos en que comportaba el deterioro de los salarios y de las condiciones de trabajo o el control obrero sobre la producción ${ }^{43}$.

$\mathrm{Si}$, por el contrario, atribuímos a los empresarios británicos la iniciativa, como hace Gospel, y no una mera actitud adaptativa a las circunstancias, los resultados son similares. De acuerdo con éste, en las etapas iniciales del proceso de industrialización del país, los empresarios británicos tomaron una serie de importantes decisiones acerca de la organización del trabajo y que el autor resume en su preferencia por operar en el mercado antes que internalizar sus relaciones con los trabajadores mediante el desarrollo de medidas de gobierno y coordinación administrativa específicas ${ }^{44}$. Aunque en su momento estas de-

${ }^{43}$ Según Jacoby (1991), pp. 173-200, la menor hostilidad de los empresarios británicos hacia los sindicatos frente a los empresarios norteamericanos, se explica, no tanto por la mayor fuerza de las organizaciones obreras en aquel pais, cuando por la existencia en Gran Bretaña de unos menores incentivos y medios para mostrar tal hostilidad que en los Estados Unidos.

44 En este punto, Gospel (1992) distingue tres áreas de actuación: «work relations», «employ- 
cisiones respondieron a una cierta racionalidad, a la larga demostraron ser poco apropiadas para garantizar la competitividad de sus empresas frente a firmas extranjeras que pudieron y supieron aplicar otras estrategias laborales ${ }^{45}$.

En Estados Unidos, por contra, el triunfo de la «moderna empresa gerencial» a finales del siglo XIX y, con ella, de la producción en serie, sería difícil de explicar sin tener en cuenta la escasez de mano de obra cualificada en el pais y la debilidad de las organizaciones obreras, afectadas no sólo ya por la hostilidad empresarial sino también por la hostilidad judicial hacia cualquier forma de acción colectiva de los trabajadores y la disposición del gobierno a emplear a la policía y al ejército para acabar con los movimientos huelguísticos.

Alli, la batalla decisiva sobre el control del trabajo y la organización de la producción tuvo lugar en los años setenta y ochenta del siglo XIX con ocasión de una serie de conflictos generales que afectaron a los sectores textil, siderúrgico y minero. Aparentemente provocados por recortes salariales, lo que de verdad estaba en juego era a quién correspondia la organización de la producción: a los trabajadores o a las empresas ${ }^{46}$. El resultado fue sistemáticamente favorable a éstas (dotadas, entre otras razones por su tamaño, de una capacidad de resistencia y de presión muy superior a las británicas) que, más rotundamente que nunca a partir de entonces se encontraron con las manos libres para impulsar la transformación del sector, lo que quiere decir, entre otras cosas, el establecimiento de sistemas de organización científica del trabajo y de nuevas políticas de personal ${ }^{47}$.

\section{III}

Los historiadores españoles de la empresa tienen ante sí la posibilidad de aprovechar el retraso acumulado en las últimas décadas con respecto a la historia empresarial anglosajona. ¿Cómo? Acometiendo una labor que no sea meramente imitativa y que tenga bien presente que el camino a recorrer no supone comenzar desde el principio ni repetir siguiendo el mismo orden los pasos dados ya con anterioridad por otras historiografías.

ment relations» e «industrial relations». Sobre el significado de cada una de ellas y lo que supone en cada caso operar en el mercado o establecer medidas internas de coordinación administrativa, véanse las pp. 3-4 y 8-10.

45 Gospel (1992), pp. 15.36.

46 Cohen (1990).

Frania y, a modo de ampliación de cuanto llevamos dicho hasta aquí sobre Gran Bretaña y Estados Unidos, véase el excelente artículo de Fridenson (1993, pp. 323-345). 
Con esta nota no hemos pretendido dar respuesta a cuanto esto supone; pero si alertar a los investigadores de nuestro país sobre la conveniencia de derribar algunos muros -especialmente, los que resultan de la excesiva compartimentación disciplinar a la que nos vemos compelidos por factores externos a la dinámica científica - y de evitar la pérdida de perspectiva glubal que supondria el que los historiadores de la empresa, por ejemplo, olvidaran que las aproximaciones macro y microeconómicas son complementarias, no excluyentes, o que fue el control del trabajo y no la eficiencia económica el factor que con más frecuencia de lo que se defiende impulsó la adopción de nuevas tecnologias de producción y condicionó las estrategias empresariales, algo en lo que han insistido de manera reiterada los historiadores del trabajo.

\section{BIBLIOGRAFÍA}

Brody, D. (1979): «The Old Labor History and The New: In Search of an American Working Class», Labor History, XX, pp. 111-126.

(1989): «Labor History, Industrial Relations, and the Crisis of American Labor», Industrial and Labor Relations Review, vol. 43, n. 1, pp. 7-18.

- (1993): «Reconciling the Old Labor History and the New», Pacific Historical Review, vol. 62, n. 1, pp. 1-18.

COHEN, I. (1990): American Management and British Labor, Westport and London, Greenwood Press.

Coleman, D. C. (1987): «The uses and abuses of business history», Business History, vol. XXIX, n. 2, pp. 141-156.

Coll, S. (1991): «Empresas versus mercados. Un boceto para una historia de la empresa», Revista de Historia Económica, año IX, pp. 263-281 y 463-478.

— y G. TORTella (1992): «Reflexiones sobre la Historia Empresarial: estado de la cuestión en Españam, Información Comercial Española, n. 708-709, pp. 13-24.

Chandler, A. J. (1984): «Comparative Business History», en D. C. Coleman and P. MaTHIAS, ed., Enterprise and History: Essays in Honour of Charles Wilson, Cambridge, Cambridge University Press.

- and R. S. TEDlow (1985): The Coming of Managerial Capitalism. A Casebook on the History of American Economic Institutions, Homewood, Richard D. Irwin.

- (1987) [1977]: La mano visible. La revolución en la dirección de la empresa norteamericana, Madrid, Ministerio de Trabajo y Seguridad Social.

(1990): Scale and Scope. The Dynamics of Industrial Capitalism, Cambridge, The Belknap Press of Harvard University Press.

CRONIN, J. E. (1989): «The "rank and file" and the social history of the working class», International Review of Social History, vol. XXXIV, pp. 62-77.

DAVIET, J. P. (1987): «Existe-t-il une business history francaise?», Annali di storia dell'impresa, núm. 3, pp. 428-484. 
Dubofsky, M. (1991): «Lost in a Fog: Labor Historians' Unrequited Search of a Synthesis», Labor History, XXXII, n. 2.

Dunlop, J. T. (1978) [1958]: Sistemas de relaciones industriales, Barcelona, Peninsula.

FINK (1991): “Intellectuals" versus "Workers": Academic Requirements and the Creation of Labor History», American Historical Review, XCVI, pp. 395-431.

FitZGERALD, R. (1988): British labour management and industrial welfare, 1846-1939, London, Croom Helm.

FontanA, J. (1987/88): «Del trabajador del campo al trabajador especializado: en torno a los Hammond», Sociología del Trabajo, nueva época, n. 2, pp. 143-151.

FRIDENSON, P. (1993): «Authority Relations in German and French Enterprises, 18801914», en J. Kocka and A. Mitchell, ed., Bourgeois Society in Nineteenth-Century Europe, Oxford, Berg, pp. 323-345.

Gordon, D. M.; EDWARDS, R. y REICH, M. (1986) [1982]: Trabajo segmentado, trabajadores divididos. La transformación bistórica del trabajo en los Estados Unidos, Madrid, Ministerio de Trabajo y Seguridad Social.

Gospel, H. F., and LitTLER, C. R. ed. (1983): Managerial Strategies and Industrial Relations. An Historical and Comparative Study, Aldershot, Gower.

- (1992): Markets, firms, and the management of labour in modern Britain, Cambridge, Cambridge University Press.

Gutman, H. (1973): «Work, Culture, and Society in Industrializing America», American Historical Review, LXXVIII, pp. 531-588.

Hobsbawm, E. (1979) [1964]: Trabajadores, Barcelona, Crítica.

- (1987) [1984]: El mundo del trabajo, Barcelona, Crítica.

JACOBY, S. M. (1991): Masters to managers. Historical and comparative perspectives on American employers, New York, Columbia University Press.

KERR, C. (1985) [1950]: Mercados de trabajo y determinación de los salarios: la «balcanización» de los mercados de trabajo, Madrid, Ministerio de Trabajo y Seguridad Social.

- (1963) [1960]: El industrialismo y el hombre industrial, Buenos Aires, EUDEBA.

Kimeldorf, H. (1991): «Bringing Unions Back In (Or Why We Need a New Old Labor History)», Labor History, XXXII, n. 1, pp. 91-129 (con respuestas de M. Kazin, A. Kessler-Harris, D. Montgomery, B. Nelson y D. Nelson y réplica del propio Kimeldorf).

Kobayashi, K., y H. Morikawa, eds. (1986): Development of managerial enterprise, Tokio, University of Tokyo Press.

Kochan, Th. A. (1980): Collective Bargaining and Industrial Relations, Irwin, Homewood, Ill.

- y otros (1993) [1986]: Las transformaciones de las relaciones Laborales en los Estados Unidos, Madrid, Ministerio de Trabajo y Seguridad Social.

KReUger, Th. A. (1971): «American Labor Historiography, Old and New: A review Essay», Journal of Social History, vol. IV, pp. 277-285.

LEE, C. H. (1990a): «Corporate behaviour in theory and history: I. The evolution of theorym, Business History, vol. 32, n. 1, pp. 17-31.

- (1990b): «Corporate behaviour in theory and history: II. The historian's perspective», Business History, vol. 32, n. 2, pp. 163-179.

LiCHT, W. (1982): «Labor economics and the labor historian», International Labor and Working Class History, vol. 21, pp. 52-62.

MARGLIN, S. (1991): «Understanding capitalism: control versus efficiency», en Bo Gus- 
tafsson, ed., Power and Economic Institutions. Reinterpretations in Economic History, Aldershot, Elgar.

MCDonell, L. T. (1991) [1984]: «“Sois demasiado sentimentales”. Problemas y sugerencias para una nueva historia del trabajom, Historia Social, n. 10, pp. 71-100.

MCKInLAY, A. (1990): «Business History and Labour History»; Labour History Review, vol. 55,3 , pp. 10-12.

McNulTY, P. J. (1980): The Origins and Development of Labor Economics, Cambridge Ma., MIT Press.

Montgomery, D. (1980): «To Study the People: The American Working Class», Labor History, vol. 21, pp. 485-512.

- (1985) [1979]: El control obrero en Estados Unidos, Madrid, Ministerio de Trabajo y Seguridad Social.

- (1987): The Fall of the House of Labor: The Workplace, the State and American Labor Activism, 1860-1925, New York, Cambridge University Press.

NakaGaWA, K., ed. (1978): Labor and Management. Proceedings of the Fourth International Conference on Business History, Tokio, University of Tokyo Press.

PALMER, G. (1983): British Industrial Relations, London, George Allen \& Unwin. and H. F. GosPeL (1993): British Industrial Relations, London, Routledge.

Piore, M., y P. Doeringer (1985) [1971]: Mercados internos de trabajo y análisis de la mano de obra, Madrid, Ministerio de Trabajo y Seguridad Social.

- y Ch. SABEL (1990) [1984]: La segunda ruptura industrial, Madrid, Alianza.

PRICE, R. (1989): «What's in a name? "Workplace history and rank and filism"», International Review of Social History, vol. XXXIV, pp. 62-77.

- (1991): «The future of the british labour history», International Review of Social History, vol. 36, pp. 249-260.

Rebitzer, J. B. (1993): «Radical Political Economy and the Economics of Labor Markets», Journal of Economic Literature, vol. XXXI, pp. 1394-1434.

Ross, S. J., and E. J. Perkins (1986): «Integrating Business History and Labor History", Business and Economic History, second series, vol. 15, pp. 43.52.

SALDERN, A. von (1994): «Current Trends in Research on the German Worker's Movement and Labor Historym; International Labor and Working Class History, 45, pp. 120-132.

SchaTZ, R. W. (1984): «Labor Historians, Labor Economics, and the Question of Synthesis», The Journal of American History, vol. 71, pp. 93-100.

SCRANTON, W. (1989): «The Workplace, Technology, and Theory in American Labor History», International Labor and Working-Class History, vol. XXXV.

Stromquist, S. (1991): «Perspectives on Labor History: The Wisconsin School and Beyond», International Labor and Working-Class History, vol. XXXIX.

THOMPson, E. P.: La formación bistórica de la clase obrera inglesa, Barcelona, Laia.

TOHARIA, L. (comp. e introd.) (1983): El mercado de trabajo: teorías y aplicaciones, Alianza, Madrid.

Tol.LIDAY, S., and J. ZeItuIn, ed. (1991): The Power to Manage? Employers and industrial relations in comparative-historical perspective, London and New York, Routledge.

TORRES VILLANUeVA, E. (1993): «La historia empresarial en España: realidades y perspectivas", San Sebastián, Actas del V Congreso de la Asociación de Historia Económica.

Valdaliso, J. M. (1993): «Algunas reflexiones acerca de la Historia Empresarial y su desarrollo en España», Revista de Historia Económica, año IX, n. 2, pp. 417.433. 
WEBB, S. y B. (1990) [1920]: Historia del sindicalismo, 1660-1920, Madrid, Ministerio de Trabajo y Seguridad Social.

WRIGHT, G. (1987): «Labor History and Labor Economics», en FIELD, A. J., ed., The Future of Economic History, Boston, Kluwer-Nijhoff Publishing, pp. 313-348.

Zeituin, J. (1987): «From labour history to the history of industrial relations», Economic History Review, 2nd ser., vol. XL, 2, pp. 159-184.

ZIEGER, R. H. (1983): «Industrial Relations and Labor History in the Eighties», Industrial Relations, vol. 22, n. 1, pp. 58-70. 